\title{
Genetic diversity of the Chinese traditional herb Blumea balsamifera (Asteraceae) based on AFLP markers
}

\author{
Y.X. Pang ${ }^{1}$, W.Q. Wang ${ }^{2}$, Y.B. Zhang ${ }^{1}$, Y. Yuan ${ }^{3}$, J.B. Yu ${ }^{1}$, M. Zhu ${ }^{1}$ and \\ Y.Y. Chen ${ }^{1}$ \\ ${ }^{1}$ Tropical Crop Genetic Resources, \\ Institute of Chinese Academy of Tropical Agricultural Sciences, \\ Danzhou, Hainan, China \\ ${ }^{2}$ College of Chinese Medical Sciences, \\ Beijing University of Traditional Chinese Medicine, Beijing, China \\ ${ }^{3}$ Environment and Plant Protection, College of Hainan University, \\ Danzhou, Hainan, China \\ Corresponding author: Y.Y. Chen \\ E-mail: chenyy1962@126.com
}

Genet. Mol. Res. 13 (2): 2718-2726 (2014)

Received August 18, 2013

Accepted November 14, 2013

Published April 14, 2014

DOI http://dx.doi.org/10.4238/2014.April.14.1

\begin{abstract}
Blumea balsamifera is a commercially important medicinal herb in China and other parts of Asia. It is used to produce borneol. This plant grows in the wild, but resources have diminished greatly in recent years. We examined the genetic diversity of this species to help develop conservation strategies; 35 plants from five provinces were analyzed using AFLPs. Eight AFLP primer combinations generated 1367 fragments, giving a mean of 172 fragments per primer combination. Polymorphism in the germplasm analysis was found for $1360(99.48 \%)$ of the fragments, of which $264(19.27 \%)$ fragments were unique (accession specific) and $423(25.33 \%)$ of the fragments were rare (present in less than $10 \%$ of the accessions). The polymorphic
\end{abstract}


fragments were used to group the accessions in a UPGMA phenogram. Most grouping was geographical. In general, accessions coming from Guizhou and Guangxi showed higher diversities as these accessions were scattered in different groups. The genetic distance estimated by Jaccard similarity coefficient index showed low variability among genotypes (coefficient value ranged from 0.60 to 0.95 ). More attention should be given to the study and conservation of the biodiversity of this economically important genus.

Key words: Blumea balsamifera; Genetic diversity; AFLP; Conservation genetics

\section{INTRODUCTION}

Blumea balsamifera is not only used as an herbal in Chinese traditional medicine but also as economic materials for L-borneol extraction, which is widely used in the cosmetic and medical industry (Donkin, 1999; State Pharmacopeia Committee of China, 2010). In contrast to synthetic borneol, borneol from B. balsamifera is non-poisonous and safer, also having a more natural aroma. In contrast to borneol from Dryobalanops aromatica Gaertn. f., it is easier to get in large amounts. Also, some other bioactive chemicals have been discovered in B. balsamifera. For example, a sesquiterpenoid isolated from its leaves has shown mild cytotoxic activity (Hasegawa et al., 2006, Norikura et al., 2008a,b), icthyothereol acetate and cryptomeridiol have exhibited moderate antifungal activities (Ragasa et al., 2005), and its methanol extracts or water extracts have shown plasmin inhibitory activity, xanthine oxidase inhibitory activity, hepatoprotective activity, etc. (Nguyen et al., 2004; Nessa et al., 2004; Osaki et al., 2005).

In recent years, due to the characteristics of rapid, accurate, polymorphism which are not affected by recessive characters, molecular markers are widely used in medicinal plant genetic diversity analysis. Li et al. (2004) used random amplified polymorphic DNA (RAPD) to study the genetic diversity of Sargentodoxa cuneata, and the results indicated that the same herbal in different areas exhibited obvious differences, where cluster analysis showed that there was a direct correlation between genetic distance and geographic distance. Zhang et al. (2010), Wu et al. (2007) and Guan et al. (2013) obtained the same results in their research of Rhodiola rosea L., Cibotium barometz (L.) J. Sm. and Gastrodia elata Blume.

B. balsamifera is found in the tropical and sub-tropical regions of Asia, especially in China and Southeast Asia. Owing to complicated and unique climate characteristics of China, after millions of years of evolution and artificial selection, B. balsamifera has gained abundant germplasm resources. These resources provided a base for developing new varieties. However, hitherto, research on $B$. balsamifera germplasm resources is still limited to the stage of morphological variation, where genetic diversity or variations revealed by molecular markers is still rare (He et al., 2005). In this study, 35 germplasm resources of $B$. balsamifera from five provinces of China were investigated by amplified fragment length polymorphism (AFLP) markers. The genotyping data were then analyzed using the unweighted pair group method with arithmetic average (UPGMA) and principal component analysis (PCA) to understand the pattern of variation. 


\section{MATERIAL AND METHODS}

\section{Plant material collection and total DNA extraction}

B. balsamifera mostly grows in semi-tropical areas of China, such as Yunnan, Hainan, Guangxi, Guizhou, and Guangdong. A total of 35 B. balsamifera individuals were collected from five provinces, according to the actual quantity in different localities. Details of collected samples are given in Table 1.

\begin{tabular}{|c|c|c|c|c|c|c|c|}
\hline \multirow[t]{2}{*}{ No. } & \multirow[t]{2}{*}{ Code } & \multirow[t]{2}{*}{ Location } & \multirow[t]{2}{*}{ Province } & \multicolumn{3}{|c|}{ Geographic Information } & \multirow[t]{2}{*}{ Appendix } \\
\hline & & & & Latitude (N) & Longitude (E) & Altitude $(\mathrm{m})$ & \\
\hline 1 & HN1 & Baisha & Hainan & $19^{\circ} 02.372^{\prime}$ & $109^{\circ} 34.146^{\prime}$ & 506.5 & Wild \\
\hline 2 & HN2 & Wanning & Hainan & $18^{\circ} 43.843^{\prime}$ & $110^{\circ} 13.591^{\prime}$ & 10.9 & Wild \\
\hline 3 & HN3 & Qiongzhong & Hainan & $18^{\circ} 99.785^{\prime}$ & $109^{\circ} 82.379^{\prime}$ & 198.0 & Wild \\
\hline 4 & HN4 & Baoting & Hainan & $18^{\circ} 68.983^{\prime}$ & $109^{\circ} 54.021^{\prime}$ & 58.0 & Wild \\
\hline 5 & HN5 & Tuichang & Hainan & $19^{\circ} 33.009^{\prime}$ & $110^{\circ} 08.832^{\prime}$ & 74.0 & Wild \\
\hline 6 & HN6 & Qionghai & Hainan & $19^{\circ} 02.456^{\prime}$ & $109^{\circ} 30.006^{\prime}$ & 210.5 & Wild \\
\hline 7 & HN7 & Wanning & Hainan & $18^{\circ} 44.843^{\prime}$ & $110^{\circ} 23.391^{\prime}$ & 20.9 & Wild \\
\hline 8 & HN8 & Wuzhishan & Hainan & $18^{\circ} 54.357^{\prime}$ & $109^{\circ} 40.258^{\prime}$ & 711.5 & Wild \\
\hline 9 & HN9 & Baisha & Hainan & $19^{\circ} 19.759^{\prime}$ & $109^{\circ} 44.335^{\prime}$ & 67.0 & Wild \\
\hline 10 & HN10 & Baisha & Hainan & $19^{\circ} 19.759^{\prime}$ & $109^{\circ} 44.335^{\prime}$ & 67.0 & Cultivar \\
\hline 11 & HN11 & Danzhou & Hainan & $19^{\circ} 42.438^{\prime}$ & $109^{\circ} 16.505^{\prime}$ & 37.5 & Cultivar \\
\hline 12 & HN12 & Diaoluo & Hainan & $18^{\circ} 78.964^{\prime}$ & $109^{\circ} 89.212^{\prime}$ & 148.0 & Wild \\
\hline 13 & YN1 & Jinghong & Yunnan & $21^{\circ} 57.704^{\prime}$ & $100^{\circ} 35.446^{\prime}$ & 1374.0 & Wild \\
\hline 14 & YN2 & Puer & Yunnan & $22^{\circ} 41.978^{\prime}$ & $100^{\circ} 56.818^{\prime}$ & 1312.0 & Wild \\
\hline 15 & YN3 & Puer & Yunnan & $22^{\circ} 43.047^{\prime}$ & $100^{\circ} 56.273^{\prime}$ & 1145.0 & Wild \\
\hline 16 & YN4 & Puer & Yunnan & $22^{\circ} 37.176^{\prime}$ & $100^{\circ} 59.661^{\prime}$ & 959.7 & Wild \\
\hline 17 & YN5 & Funing & Yunnan. & $23^{\circ} 36.690^{\prime}$ & $105^{\circ} 37.360^{\prime}$ & 818.0 & Wild \\
\hline 18 & GX1 & Qingzhou & Guangxi & $22^{\circ} 41.610^{\prime}$ & $109^{\circ} 34.143^{\prime}$ & 256.4 & Wild \\
\hline 19 & GX2 & Yulin & Guangxi & $22^{\circ} 61.401^{\prime}$ & $110^{\circ} 28.484^{\prime}$ & 256.6 & Cultivar \\
\hline 20 & GX3 & Nanning & Guangxi & $22^{\circ} 75.212^{\prime}$ & $108^{\circ} 47.625^{\prime}$ & 147.0 & Cultivar \\
\hline 21 & GX4 & Baise & Guangxi & $23^{\circ} 53.890^{\prime}$ & $106^{\circ} 32.909^{\prime}$ & 132.0 & Wild \\
\hline 22 & GX5 & Baise & Guangxi & $23^{\circ} 54.934^{\prime}$ & $106^{\circ} 28.102^{\prime}$ & 126.0 & Wild \\
\hline 23 & GZ1 & Luodian & Guizhou & $25^{\circ} 47.360^{\prime}$ & $105^{\circ} 40.535^{\prime}$ & 800.0 & Wild \\
\hline 24 & GZ2 & Xingyi & Guizhou & $24^{\circ} 52.663^{\prime}$ & $105^{\circ} 00.628^{\prime}$ & 845.0 & Wild \\
\hline 25 & GZ3 & Xingyi & Guizhou & $25^{\circ} 07.758^{\prime}$ & $105^{\circ} 55.990^{\prime}$ & 395.1 & Wild \\
\hline 26 & GZ4 & Xingyi & Guizhou & $25^{\circ} 11.031^{\prime}$ & $106^{\circ} 06.801^{\prime}$ & 690.0 & Wild \\
\hline 27 & GZ5 & Xingyi & Guizhou & $25^{\circ} 01.513^{\prime}$ & $105^{\circ} 44.089^{\prime}$ & 934.1 & Wild \\
\hline 28 & GZ6 & Xingyi & Guizhou & $25^{\circ} 07.758^{\prime}$ & $105^{\circ} 55.990^{\prime}$ & 395.1 & Wild \\
\hline 29 & GZ7 & Xingyi & Guizhou & $24^{\circ} 52.663^{\prime}$ & $105^{\circ} 00.628^{\prime}$ & 845.0 & Wild \\
\hline 30 & GZ8 & Xingyi & Guizhou & $24^{\circ} 51.910^{\prime}$ & $105^{\circ} 01.428^{\prime}$ & 805.9 & Wild \\
\hline 31 & GZ9 & Guanling & Guizhou & $25^{\circ} 55.619^{\prime}$ & $105^{\circ} 35.864^{\prime}$ & 731.5 & Wild \\
\hline 32 & GZ10 & Guanling & Guizhou & $25^{\circ} 53.425^{\prime}$ & $105^{\circ} 39.092^{\prime}$ & 656.3 & Wild \\
\hline 33 & GZ11 & Xingyi & Guizhou & $25^{\circ} 03.267^{\prime}$ & $105^{\circ} 56.235^{\prime}$ & 397.7 & Wild \\
\hline 34 & GD1 & Maoming & Guangdong & $23^{\circ} 07.973^{\prime}$ & $113^{\circ} 29.101^{\prime}$ & 68.0 & Cultivar \\
\hline 35 & GD2 & Guangzhou & Guangdong & $21^{\circ} 93.795^{\prime}$ & $111^{\circ} 35.742^{\prime}$ & 537.0 & Cultivar \\
\hline
\end{tabular}

Total genomic DNA of these materials was isolated from $0.02 \mathrm{~g}$ dry leaves using the QIAGEN DNeasy Plant Minikit according to the manufacturer instructions.

\section{AFLP analysis}

AFLP analysis was performed as previously described by Vos et al. (1995) with the following modifications: $300 \mathrm{ng}$ genomic DNA digested in $20 \mu \mathrm{L}$ with two combinations of 
enzymes ( $2 \mu \mathrm{L}$ 10X PstI buffer, 5.0 U PstI, $2.5 \mathrm{U}$ MseI and $0.2 \mu \mathrm{L} 100 \mathrm{x}$ BSA) for $16 \mathrm{~h}$, then incubated at $70^{\circ} \mathrm{C}$ for 15 min to deactivate the restriction enzymes.

PstI $(5 \mu \mathrm{mol})$ and MseI $(50 \mu \mathrm{mol})$ adaptors were ligated to the restricted DNA fragments in ligation buffer (10X $\mathrm{T}_{4}$ DNA ligase buffer, $0.2 \mathrm{U} \mathrm{T}_{4} \mathrm{DNA}$ ligase) and incubated at $16^{\circ} \mathrm{C}$ for $10 \mathrm{~h}$. All above described chemicals were obtained from TaKaRa (Dalian, China).

The diluted (10-fold) amplified products were used as the template for selective amplification. Selective amplification was carried out with nine selective primer combinations of PstI and MseI with three selective nucleotides (Table 2) in a total volume of $20 \mu \mathrm{L}$. The PCR program consisted of two segments. The first segment comprised 12 cycles, each set at $94^{\circ} \mathrm{C}$ for $30 \mathrm{~s}, 65^{\circ} \mathrm{C}$ for $30 \mathrm{~s}$, and $72^{\circ} \mathrm{C}$ for $60 \mathrm{~s}$. The annealing temperature was lowered by $0.7^{\circ} \mathrm{C}$ after each cycle during the first 12 cycles. The second segment consisted of 24 cycles set at $94^{\circ} \mathrm{C}$ for $30 \mathrm{~s}, 56^{\circ} \mathrm{C}$ for $60 \mathrm{~s}$ and $72^{\circ} \mathrm{C}$ at $60 \mathrm{~s}$ per cycle.

Table 2. Sequences of 8 AFLP selected primer combinations.

\begin{tabular}{llll}
\hline No. & Primer combinations & PstI primer $\left(5^{\prime}{ }^{\prime}-3^{\prime}\right)$ & MseI primer $\left(5^{\prime}-3^{\prime}\right)$ \\
\hline 1 & PstI-1/MseI-3 & GACTGCGTACATGCAGAA & GATGAGTCCTGAGTAACAG \\
2 & PstI-2/MseI-1 & GACTGCGTACATGCAGAC & GATGAGTCCTGAGTAACAA \\
3 & PstI-2/MseI-2 & GACTGCGTACATGCAGAC & GATGAGTCCTGAGTAACAC \\
4 & PstI-2/MseI-3 & GACTGCGTACATGCAGAC & GATGAGTCCTGAGTAACAG \\
5 & PstI-3/MseI-1 & GACTGCGTACATGCAGAG & GATGAGTCCTGAGTAACAA \\
6 & PstI-3/MseI-2 & GACTGCGTACATGCAGAG & GATGAGTCCTGAGTAACAC \\
7 & PstI-3/MseI-3 & GACTGC GTACATGCAGAG & GATGAGTCCTGAGTAACAG \\
8 & PstI-4/MseI-3 & GACTGCGTACATGCAGAT & GATGAGTCCTGAGTAACAG \\
\hline
\end{tabular}

\section{Data analysis}

Genotyping data obtained for the AFLP primer combinations was used for assessing the discriminatory power of AFLP primer combinations by evaluating three parameters: (i) polymorphism information content (PIC), (ii) marker index (MI) and (iii) resolving power (RP) (Roldán-Ruiz et al., 2000).

The PIC value for each AFLP primer combination was calculated as proposed by Roldan-Ruiz et al. (2000):

$$
\mathrm{PIC}_{\mathrm{i}}=2 \mathrm{f}_{\mathrm{i}}\left(1-\mathrm{f}_{\mathrm{i}}\right)
$$

where $P I C_{i}$ is the polymorphism information content of marker $\mathrm{i}, f_{i}$ the frequency of the marker fragments that were present and $1-f_{i}$ the frequency of marker fragments that were absent. PIC was averaged over the fragments for each primer combination.

\section{Construction of phenogram}

Prominent AFLP fragments for each primer combination were scored visually as present (1) or absent (0). Genetic similarity (GS) was estimated between pairs of accessions according to the Jaccard similarity coefficient (Jaccard, 1908) using the NTSYS-pc 2.02 software package (Rohlf, 1993). The genetic similarity matrix obtained was based on Jaccard similarity. The coefficient was used to prepare the phenogram following the unweighted pair group method with arithmetic average (UPGMA). 


\section{RESULTS}

For understanding the molecular diversity available for B. balsamifera accessions in China, a set of 35 genotypes were selected in such a way that all five provinces from where $B$. balsamifera accessions had been collected were represented. If four accessions had been collected from one town from a particular state of China, only one accession out of four accessions was selected. AFLP markers were employed for molecular profiling since AFLP provides genome-wide fingerprints.

\section{Marker polymorphism}

A total of eight primer combinations from four PstI and three MseI selective primers were used to generate AFLP profiles on 35 B. balsamifera accessions (Table 1). While analyzing the genome-wide AFLP fingerprint profiles, only prominent fragments were considered. All eight primer combinations generated a total of 1367 fragments, of which $1360(99.48 \%)$ were polymorphic and eight $(0.52 \%)$ of the polymorphic fragments were unique and accession specific (Table 3). The total number of fragments (monomorphic and polymorphic) for each assay ranged from 124 to 198 with an average of 170.9 per primer combination. The percentages of polymorphic fragments varied from 98.86 to $100 \%$ with an average of $99.48 \%$ per primer combination.

Table 3. Degree of polymorphism and information content for 8 AFLP primer combinations used.

\begin{tabular}{|c|c|c|c|c|c|c|c|c|c|}
\hline No. & Primer combinations & NTF & NMF & NPF & $\%$ Polymorphism & NUF & NRF & NSF & NSIF \\
\hline 1 & PstI-1/MseI-3 & 188 & 0 & 188 & $100 \%$ & 36 & 64 & 65 & 23 \\
\hline 2 & PstI-2/MseI-1 & 176 & 2 & 174 & $98.86 \%$ & 29 & 65 & 66 & 14 \\
\hline 3 & PstI-2/MseI-2 & 124 & 1 & 123 & $99.19 \%$ & 35 & 40 & 43 & 5 \\
\hline 4 & PstI-2/MseI-3 & 133 & 0 & 133 & $100 \%$ & 40 & 37 & 47 & 9 \\
\hline 5 & PstI-3/MseI-1 & 198 & 0 & 198 & $100 \%$ & 27 & 57 & 102 & 12 \\
\hline 6 & PstI-3/MseI-2 & 181 & 3 & 179 & $98.90 \%$ & 31 & 61 & 64 & 23 \\
\hline 7 & PstI-3/MseI-3 & 191 & 1 & 190 & $99.48 \%$ & 40 & 49 & 87 & 14 \\
\hline 8 & PstI-4/MseI-3 & 176 & 1 & 175 & $99.43 \%$ & 26 & 50 & 82 & 17 \\
\hline 9 & Total & 1367 & 8 & 1360 & $99.48 \%$ & 264 & 423 & 556 & 117 \\
\hline 10 & Minimum & 124 & 0 & 123 & $98.86 \%$ & 26 & 37 & 43 & 5 \\
\hline 11 & Maximum & 198 & 3 & 198 & $100 \%$ & 40 & 65 & 102 & 23 \\
\hline 12 & Average & 170.9 & 1 & 170 & $99.48 \%$ & 33 & 52.9 & 69.5 & 14.6 \\
\hline
\end{tabular}

$\mathrm{NTF}=$ total number of fragments generated; NMF = number of monomorphic fragments; NPF $=$ number of polymorphic fragments; NUF = total number of unique fragments; NRF = total number of rare fragments; NSF = total number of shared fragments; NSIF $=$ total number of similar fragments.

Marker informativeness for the eight AFLP primer combinations was analyzed using several parameters (Table 3). The fragments generated by the eight primer combinations varied from 124 (PstI-2/MseI-2) to 198 (PstI-3/MseI-1) with an average of 170.9 per primer combination, and percentage of polymorphic fragments varied from $98.48 \%$ (PstI-3/MseI-3) to $100 \%$ with an average of $99.48 \%$ per primer combination. The unique fragments (NUF), present in only one accession for the given primer combination, varied from 26 (PstI-4/MseI3 ) to 40 (PstI-3/MseI-3) with a total of 264 eight primer combinations.

AFLP fragments observed in less than $10 \%$ of the accessions with a given primer combination were considered rare fragments (NRF). A total of 423 rare fragments were observed with an average of 52.9 rare fragments per primer combination (Table 3). Highest number of 
NRF (65) was generated by the primer combination of PstI-2/MseI-1, followed by 61 NRF generated by primer PstI-3/MseI-2. The fewest NRF (37) were generated by the primer PstI-2/ MseI-3. Shared fragments (NSF) were those that were scored in $70 \%$ of accessions. A total of 556 shared fragments were observed ranging from 43 (PstI-2/MseI-2) to 102 (PstI-3/MseI-1). Similar fragments (NSIF) were the fragments present in more than $70 \%$ of accessions, where eight AFLP primer combinations in total generated 117 similar fragments with an average of 14.6 per primer combination.

\section{AFLP features}

PIC, MI (a feature of a marker and therefore MI calculated for all primer combinations) and RP (a feature of the primer combination that indicates the discriminatory potential of the primer combination) are three important indices of DNA molecular markers. These indices were analyzed with POPGENE version 1.31 (Yeh et al., 1999). The PIC value of 1360 polymorphic fragments ranged from 0.05 to 0.50 , with an average of 0.1914 per primer combination. MI ranged from 19.57 to 50.81 with an average of 33.08 per primer combination. The highest value (50.81) was scored with PstI-3/MseI-1 primer pair and the minimum value (19.57) for PstI-2/MseI-2 (Table 4). A positive correlation was observed between MI and PIC values $\left(\mathrm{r}^{2}=0.78, \mathrm{P}<0.005\right)$. RP ranged from 37.20 to 75.87 with an average of 58.31 per primer combination. The highest value (75.37) was found in the primer combination for PstI3/MseI-1 and the lowest value (37.20) for PstI-2/MseI-2 (Table 4). RP values were found in positive correlation with $\mathrm{MI}\left(\mathrm{r}^{2}=0.93, \mathrm{P}<0.005\right)$.

\begin{tabular}{|c|c|c|c|c|c|}
\hline No. & Primer combinations & PIC & EMR & MI & $\mathrm{RP}$ \\
\hline 1 & PstI-1/MseI-3 & 0.1813 & 188 & 34.08 & 56.86 \\
\hline 2 & PstI-2/MseI-1 & 0.1860 & 174 & 32.36 & 58.57 \\
\hline 3 & PstI-2/MseI-2 & 0.1591 & 123 & 19.57 & 37.20 \\
\hline 4 & PstI-2/MseI-3 & 0.1626 & 133 & 21.63 & 37.31 \\
\hline 5 & PstI-3/MseI-1 & 0.2566 & 198 & 50.81 & 75.37 \\
\hline 6 & PstI-3/MseI-2 & 0.1798 & 179 & 32.18 & 67.60 \\
\hline 7 & PstI-3/MseI-3 & 0.1993 & 190 & 37.87 & 69.49 \\
\hline 8 & PstI-4/MseI-3 & 0.2066 & 175 & 36.16 & 64.11 \\
\hline 9 & Minimum & 0.1591 & 123 & 19.57 & 37.20 \\
\hline 10 & Maximum & 0.2566 & 198 & 50.81 & 75.37 \\
\hline 11 & Average & 0.1914 & 170 & 33.08 & 58.31 \\
\hline
\end{tabular}

$\mathrm{PIC}=$ polymorphism information content $\mathrm{EMR}=$ effective multiplex ratio; $\mathrm{MI}=$ marker index; $\mathrm{RP}=$ resolving power.

\section{Genetic diversity and phylogenetic analysis}

Genotyping data obtained for all 1360 polymorphic fragments from eight primer combinations were used to estimate pairwise similarity comparisons between the accessions. The genetic similarity matrix was determined using the Jaccard similarity coefficient using the NTSYS-pc program. Subsequently, the genetic similarity matrix was used for constructing the UPGMA phenogram with the help of the NTSYS-pc program. The similarity coefficient values of the phenogram ranged from 0.66 to 0.91 , suggesting a broad genetic base (Figure 1). 


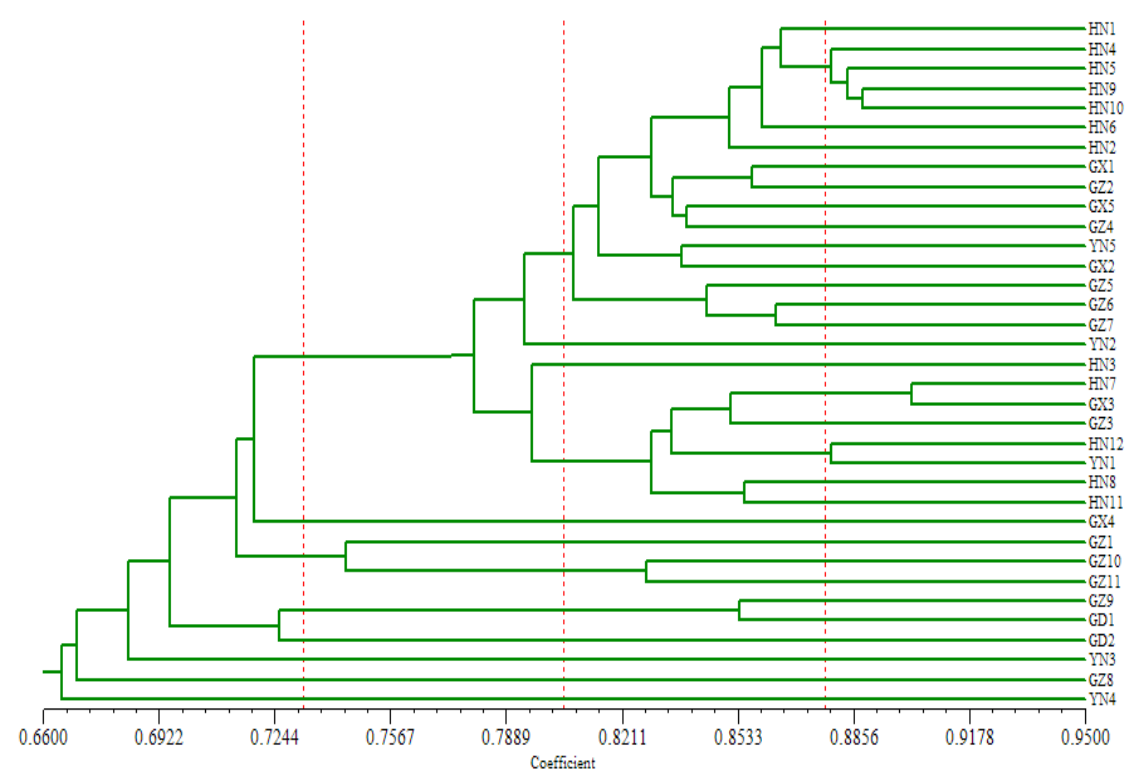

Figure 1. Dendrogram of 35 Blumea balsamifera accessions based on AFLP analysis using UPGMA.

On the basis of the UPGMA cluster diagram (Figure 1), all accessions were classified into four major clusters (Cluster I, II, III and IV). Cluster I contained 18 accessions, with a coefficient of 0.7953 , and could be divided into four subclusters. Subcluster "i" contained seven accessions from Hainan (HN1, HN2, HN4, HN5, HN6, HN9 and HN10), with a coefficient of 0.8533. Subcluster "ii" contained four accessions, with two accessions from Guangxi (GX1 and GX5) and the others from Guizhou (GZ2 and GZ4), with a coefficient of 0.8339. Subcluster "iii" contained two accessions, from Yunnan (YN5) and Guanxi (GX2), respectively. Subcluster "iv" contained three similar accessions from Guizhou (GZ5, GZ6 and GZ7) with a coefficient of 0.8469. Excluding those subclusters, one accession from Yunnan (YN3) also belonged to Cluster I.

Cluster II contained eight accessions. Five of them were from Hainan (HN3, HN7, HN8, HN11 and HN12), and the others were from Guangxi (GX3), Yunnan (YN1) and Guizhou (GZ3), respectively. In this cluster, except for HN3, all other accessions showed a high degree of homology ( $85 \%$ similarity). Cluster III contained three accessions from Guizhou (GZ1, GZ10 and GZ11), with a coefficient of 0.7349 . Cluster IV also contained three accessions, one from Guizhou (GZ9) and the other two from Guangdong (GD1 and GD2).

Excluding those accessions of four major clusters, there were also three accessions, two of them from Yunnan (YN2 and YN4) and the other from Guizhou (GZ8). These accessions showed a low degree of homology $\left(0.6664^{\sim} 0.6988\right)$ with the accessions classified into four major clusters. These results indicated that those accessions would be important germplasm resources for hereditary improvement of cultivated B. balsamifera.

\section{DISCUSSION}

In the case of B. balsamifera, there are many chemical constituents and various phar- 
macological studies have been carried out, but few studies have been conducted to assess molecular diversity and germplasm collection. Compared to RAPD, inter-simple sequence repeats, restriction fragment length polymorphism and microsatellites, AFLP is an informationrich marker system due to its ability to generate a large number of polymorphic/informative loci simultaneously in a single lane with a single-primer combination, it has been successfully used to investigate the diversity in several tropical plant species, such as Hevea, cassava, etc. In our research, the AFLP method was chosen to assess the genetic similarity of B. balsamifera from five provinces of China.

The efficiency of a molecular marker technique depends upon the amount of polymorphism it can detect among the set of accessions under investigation. High quality marker profiling was obtained with eight AFLP primer combinations in 35 accessions collected from provinces of China. All primer combinations used were found to be polymorphic and yielded 1360 of 1367 (99.48\%) polymorphic fragments. The level of polymorphism in the present study was higher compared to another AFLP diversity study (88.2-97.2\%), each assay ranged from 124 to 198 with an average of 170.9 per primer combination. Also, the AFLP features PIC, MI and RP were determined. PIC ranged between 0.05 and 0.50 with an average of 0.1914 per primer combination, MI ranged from 19.57 to 50.81 with an average of 33.08, and RP ranged from 37.20 to 75.87 with an average of 58.31, A positive correlation was observed between RP, MI and PIC $\left(\mathrm{r}^{2}=0.93, \mathrm{r}^{2}=0.78\right)$.

Genetic similarity between 35 accessions was calculated using the NTSYS-pc program with the Jaccard similarity coefficient. The results showed that similarity coefficient values of the phenogram ranged from 0.66 to 0.91. Excluding two accessions from Yunnan (YN2 and YN4) and one accession from Guizhou (GZ8), other accessions could be classified into four major clusters (Cluster I, II, III and IV). Excluding those accessions of four major clusters, there were also three accessions, two of them from Yunnan (YN2 and YN4) and one from Guizhou (GZ8), which showed a low degree of homology (0.6664-0.6988) with those accessions of the four major clusters.

In summary, the present study provided a larger number of reliable and reproducible fingerprint profiles for an elite collection of 35 accessions of $B$. balsamifera collected from five provinces of China. On the one hand, similarity analysis of $B$. balsamifera showed lower genetic diversity and higher similarity of $B$. balsamifera, and this included the necessity of protecting the germplasm resources of $B$. balsamifera. On the other hand, higher genetic diversities were documented in the accessions of Hainan and Guizhou. The availability of unique or rare fragments present in different accessions together with genetic dissimilarly data would be very useful for improvement of the species through conventional breeding methods as well as molecular breeding approaches such as marker assisted selection.

\section{ACKNOWLEDGMENTS}

Research supported by the National Natural Science Foundation of China (\#30860370) and the National Natural Science Foundation of Hainan (\#311023).

\section{REFERENCES}

Donkin RA (1999). Dragon's Brain Perfume: an Historical Geography of Camphor. Brills Indological Library, 74-78. Guan P, Shi JM and Chen F (2013). AFLP analysis of Gastrodia elata B1 from different regions. J. Plant Genet. Resour. 
14: 66-73.

Hasegawa H, Yamada Y, Komiyama K, Hayashi M, et al. (2006). Dihydroflavonol BB-1, an extract of natural plant Blumea balsamifera, abrogates TRAIL resistance in leukemia cells. Blood 107: 679-688.

He YN, Ding Y and Xian FR (2005). Observation on germplasm variation structure and selection of Blumea balsamifera. Guizhou Agr. Sci. 33: 33-36.

Jaccard P (1908). Nouvelles recherches sur la distribution florale. Bull. Soc. Vaud. Sci. Nat. 44: 223-270.

Li JM, Jin ZX and Zhong ZC (2004). RAPD analysis is of genetic diversity of Sargentodoxa cuneata at different altitude and the influence of environmental factors. Acta Ecol. Sin. 24: 567-573.

Nessa F, Ismail Z, Mohamed N and Haris MRHM (2004). Free radical-scavenging activity of organic extracts and of pure flavonoids of Blumea balsamifera DC leaves. Food chem.. 88: 243-252.

Nguyen MT, Awale S, Tezuka Y, Tran QL, et al. (2004). Xanthine oxidase inhibitory activity of Vietnamese medicinal plants. Biol. Pharm. Bull. 27: 1414-1421.

Norikura T, Kojima-Yuasa A, Shimizu M, Huang X, et al. (2008a). Mechanism of growth inhibitory effect of Blumea balsamifera extract in hepatocellular carcinoma. Biosci. Biotechnol. Biochem. 72: 1183-1189.

Norikura T, Kojima-Yuasa A, Shimizu M, Huang X, et al. (2008b). Anticancer activities and mechanisms of Blumea balsamifera extract in hepatocellular carcinoma cells. Am. J. Chin. Med. 36: 411-424.

Osaki N, Koyano T, Kowithayakorn T, Hayashi M, et al. (2005). Sesquiterpenoids and plasmin-inhibitory flavonoids from Blumea balsamifera. J. Nat. Prod. 68: 447-449.

Ragasa CY, Co AL and Rideout JA (2005). Antifungal metabolites from Blumea balsamifera. Nat. Prod. Res. 19: 231-237. Rohlf FJ (1993). NTSYS-pc (Numerical Taxonomy and Multivariate Analysis System). Version 2.1, Exeter Software, Setauket.

Roldán-Ruiz I, Dendauw J, Van Bockstaele E and Depicker A (2000). AFLP markers reveal high polymorphic rates in ryegrasses (Lolium spp.). Mol. Breed. 6: 125-134.

State Pharmacopeia Committee of China (2010). Pharmacopoeia of the People's Republic of China (PPRC). People's Medical Publishing House, Beijing.

Vos P, Hogers R, Bleeker M, Reijans M, et al. (1995). AFLP: a new technique for DNA fingerprinting. Nucleic Acids Res. 23: 4407-4414.

Wang XL, Song JX and Li BZ (2009a). Primary study of the relationship between the SSR of Gastrodia elata Blume and the gastrodin content. W. Sci. Technol. (Modernization of Traditional Chinese Medicine and Materia Medica) 11: 880-884.

Wang Q, Ruan X, Jiang H, Meng Q, et al. (2009b). Genetic diversity of different geographical populations of Rhodiola rosea based on AFLP markers. Zhongguo Zhong. Yao Za Zhi. 34: 2279-2284.

Wu L, Deng HP and Xu J (2007). Genetic diversity of Cibotium barometz (L.). J. Sm. by AFLP Anal. China J. Chin. Mat. Med. 32: 1467-1468.

Yeh FC, Yang RC and Boyle T (1999). Available at [http://www.ualberta.ca/ fyeh/]. Accessed October 24, 2011.

Zhang YH, Hou Y and Lou AR (2010). Population genetic diversity of Rhodiola dumulosa in Northen China inferred from AFLP markers. Chin. J. Plant Ecol. 34 1084-1094. 